\title{
Modelling, Evaluation and Simulation during the Early Design Stages: Toward the Development of an Approach Limiting the Need for Specific Knowledge
}

\author{
Galina Medyna $^{a}{ }^{*}$, Sarayut Nonsiri ${ }^{a}$, Eric Coatanéa $^{a}$, and Alain Bernard ${ }^{b}$ \\ ${ }^{a}$ Department of Engineering Design and Production, Aalto University \\ School of Engineering, Espoo, Finland \\ ${ }^{b}$ IRCCYN UMR CNRS 6597, Ecole Centrale Nantes, Nantes, France
}

\begin{abstract}
The early stages of the design process are keys in the development of products and services. Nevertheless, they are marked by multiple constraints imposed on them, such as, most notably, a limited amount of time available for modelling and evaluating ideas and concepts. The present article develops an approach for modelling, and simulating initial design solutions during these critical early stages. The final objective is to minimize the amount of prerequisite knowledge a designer should have on the artefact being designed in order to propose, develop, and evaluate early models. First, the current work analyses the conditions necessary to develop a modelling and comparison environment for early design solutions. This is done through mathematical considerations of the design process. In a second part, the work proposes a modelling and simulation approach and develops the machinery behind it. The approach integrates and maps a series of normalized semantic descriptions of functions, generic engineering components and variables, a set of elementary laws associated with these components, and a set of elementary base units. All these elements are used to refine and guide the modelling process. This process is uses the Vaschy-Buckingham theorem followed by an approximation of the generic law describing the general behaviour of elementary components. This combination leads to an approximated model of the behaviour of the studied artefact. The model is further developed by implementing the behaviour in a system dynamics tool using two basic bricks of the system dynamics language, converters and flows. In a final part, the approach is illustrated through the case study of a beam structure.
\end{abstract}

Keywords: early design, modelling, simulation, evaluation models, dimensional analysis, system dynamics, topology

\section{Introduction}

Products and services that we use every day are the result of a multi-faceted and often multidisciplinary design process. This process is growing all the more complex as artefacts face more and more constraints, from needs to streamline manufacturing to taking environmental issues into consideration. In recent decades, the design process has been the subject of scientific research and, although multiple

* Corresponding author. E-mail: galina.medyna@aalto.fi 
representations exist, a common backbone of phases stands out - conceptual, embodiment, and detailed design (Motte, et al., 2011). Multiple studies indicate that over $80 \%$ of the life-cycle costs of a product are committed during the early design phases (e.g. Lotter, 1986; Hsu \& Woon, 1998; Stasinopoulos, et al., 2009). Indeed, decisions made at the conceptual design stage have a significant influence on such aspects as cost, performance, reliability, safety, or environmental impact of an artefact; a poorly conceived design concept can never be compensated by a good detailed design. In recent years, companies, researchers, and authorities have come to understand that in order to remain competitive and meet increasingly complex customer requirements and demands there are fundamental benefits to properly managing the design process and actively focusing on conceptual design(Pahl \& Beitz, 1984; Dardy, et al., 2003; Cross, 2007).

Several methods, tools, and techniques have been developed to support the various phases of design, ranging from design guidelines aimed at specific fields to creativity techniques used to promote innovative solutions. Increasingly, attention has been directed towards the development of support for conceptual-level design activities and of methodologies that can be used during the conceptual stage of the design process. Nevertheless, these attempts remain partially unsatisfactory due to the fact that knowledge during this early phase of a product's life cycle is usually incomplete and approximate (i.e. design requirements and constraints are far from being fully defined). In order to deal with such uncertainty, designers often choose to restrict themselves to narrow, well-defined sub-problems and subtasks that should lead to a sound global design but effectively often lead to sub-optimal overall solutions and trial and error approaches (Sydenham, 2004).

The present research work aims to provide an analytical tool for the early stages of the design process that addresses the issues that exist in other methods and tools. In order to be accessible, the practical methodology developed should remain simple while still relying on sound scientific principles. The scope of this article is the conceptual design activity (French, 1999; Yannou, 2000), as represented in Fig. 1, and more specifically Step 3 - the evaluation of concepts of solutions. First, though, some aspects of the refinement of the initial definition of the needs into a functional definition of the problem are considered (Ulrich \& Eppinger, 2000; Miled, 2003); this is represented as Step 1 in Fig. 1 - problem clarification and formulation. The focus proper is the creation of a generic modelling and simulation framework that can be applied to the evaluation of different requirements (e.g. functional definition of needs in Fig. 1). This model exhibits similarities with the recursive vision of Zeng (Zeng, 2008).

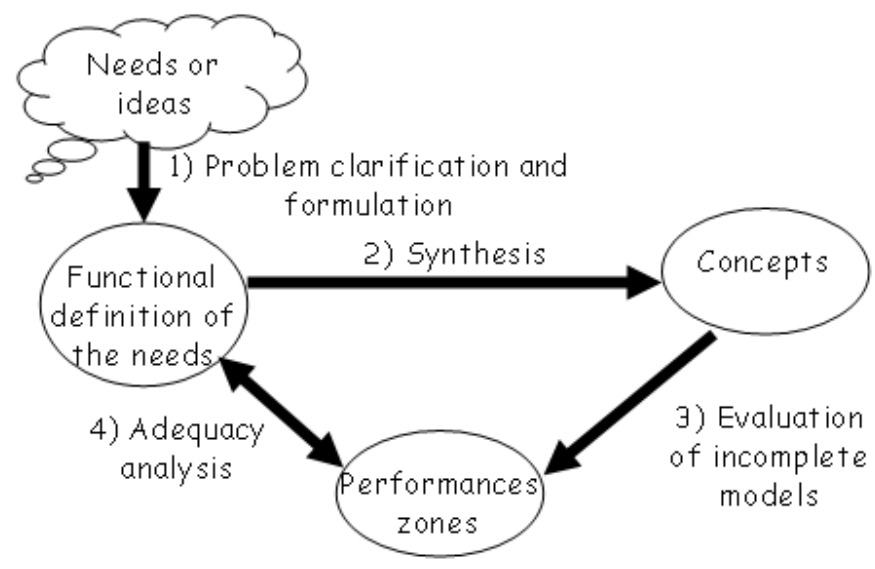

Fig. 1. The conceptual design stage, based on (Yannou, 2000).

The present work is structured as follows. At first, Section 2 demonstrates that a transitional step is needed between the functional representation of the design problem and the synthesis of the initial design concepts. For this purpose, the General Design Theory framework (GDT) and the characteristics of the most appropriate topological structure needed to compare concepts of solutions are considered; a topologic metric space best suited for comparisons between solutions is created. The latter part of Section 
2 describes step by step the proposed methodology. This methodology provides the analysis stages needed to develop a representation of concepts of solutions in the form of graph models of a component that can be simulated. Section 3 illustrates the proposed framework using the case study of the selection of the cross section shape of a beam supporting measuring instruments. Finally, Section 4 concludes on the work and future expansions of the methodology.

\section{A Graph-Based Representation Based on Dimensional Analysis for System Components}

The General Design Theory (GDT) (Yoshikawa, 1981; Tomiyama \& Yoshikawa, 1987) is used as the base structure for the present article because, as stated by Reich (Reich, 1995), "GDT is a notable exception in the domain of design theory in the sense that it is a mathematical theory of design." The main hypothesis of GDT consists in stating that design has a topological structure and therefore geometrical attributes. From this point of view all design is strongly linked to geometry. Nevertheless, the scope of topology is not limited to these geometrical considerations and is broader; it could be seen as an extension of the concept of continuity (Reich, 1995; Sutherland, 1975). The major impact of this type of viewpoint is that topology can then be considered as exhibiting the properties linked to the concept of continuity, the four major ones being, according to Reich (Reich, 1995) and Bourbaki (Bourbaki, 1966), distance, continuity, convergence, and transformation.

The notion of distance between two functions or two product concepts is interesting during the selection and evaluation process of conceptual design. It is a metric that can provide insight into such aspects as how close two functions or concepts of solutions are, or how far some concepts of solutions from the expected requirements. Continuity guarantees that a small change in the functional description will result in a small change in the product concepts and vice versa. This property also ensures that a mapping is possible between functions and attributes that describe product concepts. Convergence oversees that a sequence of small incremental changes in product concept attributes will only cause small incremental changes to their functionality and vice versa. Transformation is a property that guarantees that any transformation from a space, model, etc. to another conserves continuity and convergence.

Further continuing along this analogy between topology and continuity, the next step is to define the elementary properties that a design problem should exhibit in order to support these four properties of distance, continuity, convergence and transformation when moving from a functional representation to a physical implementation.

The notion of distance has been investigated in the research work of Coatanéa (Coatanéa, 2005). He has established several necessary conditions to obtain a classification space, which is the first step necessary to later form a metric space. This metric space is, according to GDT, the best topological space to compare concepts. The three fundamental conditions necessary for a classification space are:

- a fundamental system of entourages,

- a sufficiently detailed fundamental system of entourages in order to ensure separation,

- a countable fundamental system of entourages.

In a more practical manner, the first condition of metrisation, having a fundamental system of entourages, means that a set of generic engineering concepts is needed. Concepts, in this case, are a set of elements, a detailed presentation for which can be found in Coatanéa (Coatanéa, 2005). To describe this set of generic engineering concepts, there is a need for a normalized functional vocabulary; a partial reproduction of one is shown in Table 1, as adapted from Hirtz et al. (Hirtz et al., 2002). Furthermore, fundamental types of variables describing a system need to be established. Flow, effort, displacement, and momentum are generic variables used in Bond Graph theory (Top, 1993), to this initial set a fifth type of variables, named connecting variables, is added (Coatanéa, 2005). In physics, for example, a connecting variable is the Young modulus, $E$. 
Table 1. Partial representation of the reconciled functional taxonomy adapted from Hirtz (2002)( entire table available in Coatanéa (2005))

\begin{tabular}{|c|c|}
\hline \multicolumn{2}{|c|}{ Domains: Physical, economical, information } \\
\hline Power variables class & State variables class \\
\hline $\begin{array}{c}\text { Generalized effort }(e), \text { and } \\
\text { generalized flow }(f), \\
\text { And connecting variables }\end{array}$ & $\begin{array}{l}\text { Generalized displacement }(q), \\
\text { generalized momentum }(p), \text { and } \\
\text { connecting variables }\end{array}$ \\
\hline \multicolumn{2}{|c|}{$\begin{array}{l}\text { Open systems mechanisms, command unit mechanisms and signal } \\
\text { mechanisms }\end{array}$} \\
\hline $\begin{array}{r}6 \text { basic organs families } \\
\text { Source o } \\
\text { D } \\
\text { 1-org } \\
\text { Rotation } \\
\text { Sphe } \\
\text { Li } \\
\text { C } \\
\end{array}$ & $\begin{array}{l}\text { lementary organs and laws } \\
\text { nism: } \\
\text { mechanisms: } \\
\text { nism: } \\
\text { nisms: } \\
\text { lon- organ } \\
\text { on-organ } \\
\text { an } \\
\text { rgan } \\
\text { gan } \\
\text { rgan } \\
\text { al link-organ } \\
\text { ink-organ } \\
\text { gan } \\
\text { n } \\
\text {-organ } \\
\text { an } \\
\text { an } \\
\text { isms: } \\
\text { nan }\end{array}$ \\
\hline
\end{tabular}


Table 2 below provides provides an example of generic terms as extracted from the Hirtz taxonomy from the Hirtz et al. (Hirtz et al., 2002) taxonomy. Such a table can help name the generic variables involved in a certain technology used to implement a certain type of solution to a design problem. Depending on the field of domain, these generic variables are referred to under different names; Table 3 and Coatanéa (Coatanéa, 2005) list several examples of such differences.

Table 2. Partial classifications of domains and related names for state variables (adapted from Hirtz (2002))

\begin{tabular}{|c|c|c|c|c|}
\hline \multicolumn{5}{|c|}{ Taxonomy of the functions } \\
\hline $\begin{array}{l}\text { Possible } \\
\text { Domains }\end{array}$ & $\begin{array}{c}\text { Primary } \\
\text { Function } \\
\end{array}$ & $\begin{array}{c}\text { Secondary } \\
\text { Function } \\
\end{array}$ & $\begin{array}{c}\text { Tertiary } \\
\text { Function }\end{array}$ & Correspondences \\
\hline \multirow{4}{*}{ 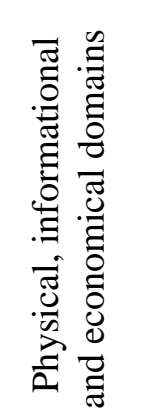 } & \multirow[t]{4}{*}{ Branch } & \multirow[t]{4}{*}{ Separate } & \multirow{4}{*}{$\begin{array}{l}\text { Divide } \\
\text { Extract } \\
\text { Remove }\end{array}$} & Isolate, sever, disjoin \\
\hline & & & & $\begin{array}{c}\text { Detach, isolate, release, sort, split, disconnect, } \\
\text { subtract }\end{array}$ \\
\hline & & & & Refine, filter, purify, percolate, strain, clear \\
\hline & & & & Cut, drill, lathe, polish, sand \\
\hline
\end{tabular}

A conceptual solution can be implemented using different elementary components classified in fundamental families. The basic set of families is formed by the Bond Graph families (Shim, 2002); it should be expanded using a set of other elementary types of components such as transformation organs (Coatanéa, 2005). The connection between the elementary functions and organs is done via a mapping table, as represented in Table 2 . This table not only provides a mapping between generic components but also the nature of the generic variables involved in the organs and the causality between them. The behaviour of the generic organs is governed by generic laws, most of which are derived from Bond Graph theory and Coatanéa's (Coatanéa's, 2005) work.

Fig.2 represents an example of a generic law associated with a C-organ (e.g. Capacitor organ), a storage organ mechanism.

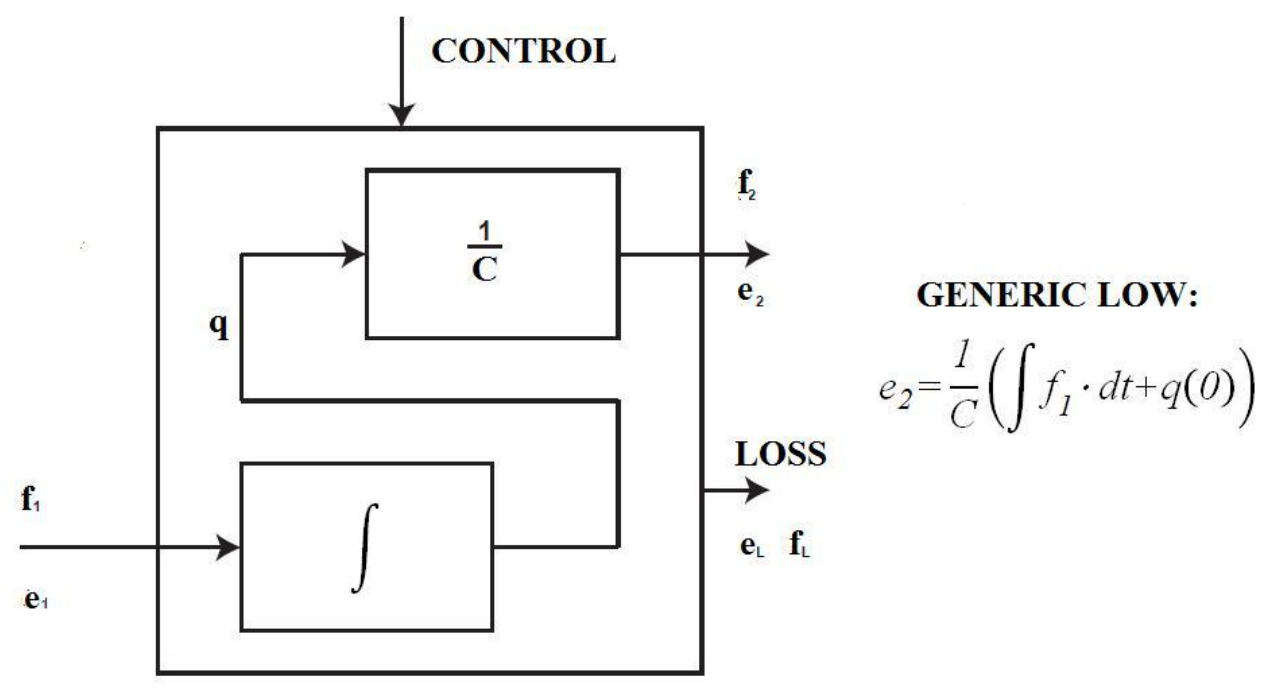

Fig. 2. SADT representation of a C-organ. 
Table 3. Classification of elementary variables and organs

\begin{tabular}{|c|c|c|c|c|c|}
\hline Domain & Energy & Primary fields & "Secondary fields & $\begin{array}{c}\text { Generalised } \\
\text { displacement }(q)\end{array}$ & $\begin{array}{c}\text { Generalised } \\
\text { momentum (p) }\end{array}$ \\
\hline \multirow[t]{28}{*}{ Physical } & Energy & $\begin{array}{l}\text { Mechanical } \\
\text { translation }\end{array}$ & & Displacement & "Momentum \\
\hline & & Mechanical & & Angular & Angular \\
\hline & & Rotational & & Displacement & Momentum \\
\hline & & Electrical & & Electrical charge (q) & $\begin{array}{l}\text { Flux } \\
\text { Linkage (OR) } \\
\text { Magnetic flux }\end{array}$ \\
\hline & & Pneumatic & & Volume & Pressure momentum \\
\hline & & Hydraulic & & Volume & Pressure \\
\hline & & & & & Momentum \\
\hline & & Thermodynamic & Heat exchange & Entropy (S) & Temperature \\
\hline & & & & & Momentum \\
\hline & & & Mass flow & Mass & Free Gibbs enthalpy \\
\hline & & AND & & & Momentum \\
\hline & & Environmental & & & \\
\hline & & & Mechanical & Volume & Pressure \\
\hline & & & Work & & Momentum \\
\hline & & Magnetic & & Displacement & $\begin{array}{l}\text { Magnetic } \\
\text { momentum }\end{array}$ \\
\hline & & Acoustic & & Volume & $\begin{array}{l}\text { Particle pressure } \\
\text { momentum }\end{array}$ \\
\hline & & Biological & & Volume & Pressure momentum \\
\hline & & Chemical & & Volume & Pressure momentum \\
\hline & & Electromagnetic & & Wave length $(\lambda)$ & Momentum \\
\hline & & & Gamma (photon $\left.+\Delta \lambda_{1}\right)$ & Wave length $(\lambda)$ & Momentum \\
\hline & & & X-Ray (photon +2 neutrons) & Wave length $(\lambda)$ & Momentum \\
\hline & & & $\mathrm{UV}\left(\right.$ photon $\left.+\Delta \lambda_{2}\right)$ & Wave length $(\lambda)$ & Momentum \\
\hline & & & Visible (photon $\left.+\Delta \lambda_{3}\right)$ & Wave length $(\lambda)$ & Momentum \\
\hline & & & Infra-red (photon $\left.+\Delta \lambda_{4}\right)$ & Wave length $(\lambda)$ & Momentum \\
\hline & & & Radio (atoms, molecules + ) & Wave length $(\lambda)$ & Momentum \\
\hline & & Radioactivity & & Displacement & Momentum \\
\hline & & & $\begin{array}{l}\text { Beta (electron }+ \text { antineutrino } \\
\text { or position }+ \text { neutrino) }\end{array}$ & Displacement & Momentum \\
\hline & & & Alpha (proton +2 neutrons) & Displacement & Momentum \\
\hline \multirow[t]{7}{*}{ Information } & Signal & Status & Auditory & Information & Information flux \\
\hline & & & Olfactory & Charge (Iq) & Linkage \\
\hline & & & Tactile & & \\
\hline & & & Taste & & \\
\hline & & & Visual & & \\
\hline & & Control & Analog & Information & Information flux \\
\hline & & & Discrete & Charge (Iq) & Linkage \\
\hline \multirow[t]{3}{*}{ Economical } & Monetary & Control & Physical currency & Monetary & Monetary flux \\
\hline & & & Digital currency & Charge (Eq) & \\
\hline & & & Exchange & & \\
\hline
\end{tabular}


Table 4. Mapping between mechanism families, organs, functions, fields, power and state variables

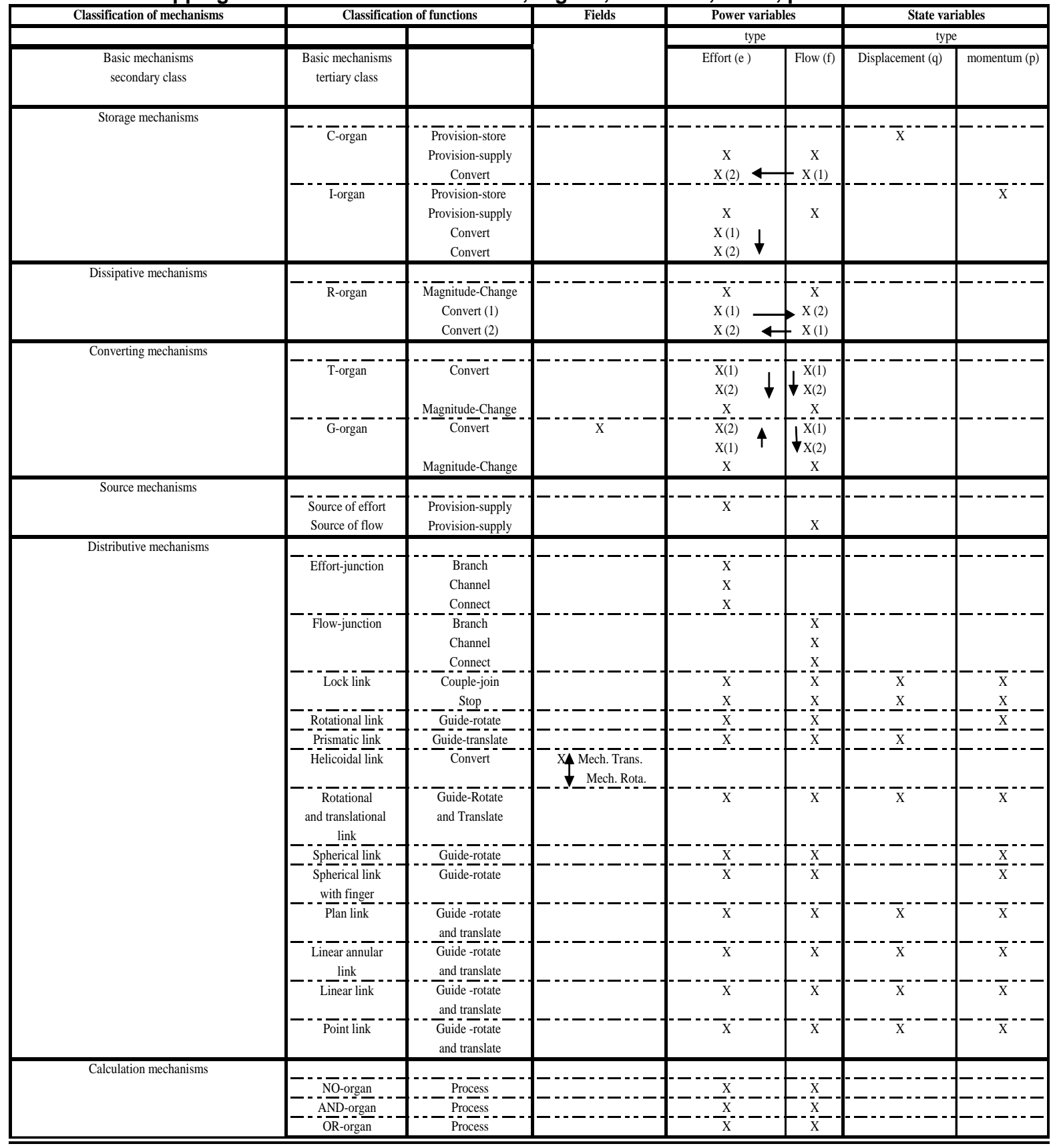

The second condition of metrisation listed above consists of ensuring that the system of entourages is detailed enough that there is separation between all the concepts of solutions. The property of separation is deeply rooted in GDT (Yoshikawa, 1981; Tomiyama \& Yoshikawa, 1987) through the axiom of separation/recognition. In the present work this property of separation is obtained through the set of basic elementary concepts described briefly above, and more extensively described in Coatanéa (Coatanéa, 2005).

The third condition of metrisation is related to the countable characteristic of the set of concepts verifying the first condition. To be countable a set of concepts should be based on a fundamental system of quantities, taken from the SI system of units augmented by two other basic metrics, one for information 
content and another for economic exchange (Sonin, 2001; Coatanéa, 2005). Table 5 lists the different elementary metrics that should be used to verify this third condition.

Table 5. Elementary set of metrics

\begin{tabular}{lcc}
\hline \multicolumn{3}{c}{ The seven Base SI quantities and units } \\
\hline \hline Physical quantity (symbol) & Base unit & Unit Symbol \\
\hline Length (L) & meter & $\mathrm{m}$ \\
Time (T) & second & $\mathrm{s}$ \\
Mass (M) & kilogram & $\mathrm{kg}$ \\
Electric current (A) & ampere & $\mathrm{A}$ \\
Thermodynamic & kelvin & $\mathrm{K}$ \\
temperature (K) & candela & $\mathrm{cd}$ \\
Luminous intensity (Cd) & mole & $\mathrm{mol}$ \\
Amount of substance (Mol) & The two non-physical quantities and units \\
\hline \multicolumn{2}{c}{ Base unit } & Unit Symbol \\
\hline Quantity (symbol) & shannon & Sh \\
Informational (Sh) & cost & $€$ or $\$$ or others \\
Economical (C)
\end{tabular}

A classification space is obtained when the three conditions detailed above are validated. This classification space can be transformed into a metric space using the Vaschy-Buckingham theorem (Barenblatt, 1979; Sonin, 2001) and the machinery introduced Bashkar and Nigam (Bhashkar \& Nigam, 1990). This transformation holds the premises for integrating dimensional analysis theory (DAT) (Matz, 1959; Barenblatt, 1979; Sonin, 2001) into the design approach proposed further in this work. It should be noted that the approach developed is compliant with the vision of the design activity traditionally developed in engineering literature (Otto \& Wood, 2001; Pahl \& Beitz, 1984; NASA, 2007). In both cases, a set of elementary concepts is used to transform and enrich the initial material of any development project (e.g. initial needs). It is then progressively refined by turning that material into requirement models that are directly used by engineers to make a synthesis of design solutions. These initial design solutions should be compared and evaluated (Fig. 1) to further develop the most promising ones; this might be very challenging if the solutions are implemented using radically different technologies assessed by different performance metrics.

Nevertheless, even though different technologies can be used to implement a function, the inputs and outputs of each solutions will have similar properties (e.g. at the early design stage, the variables and metrics will be the same). Consequently, the difference between different solutions lies in the nature of the technology and what is present inside the box describing the solution. Multiple box models exist in system engineering literature (NASA, 2007), such as functional boxes associated with inputs and outputs representing "what is required to be developed" or solution side boxes representing "how the solution is implemented". In this second category, a distinction can be made between a white box, where the contents of the box are known in details, a grey box, where the knowledge is partial, and a black box, where what is inside the box is unknown.

In the context of this work, there is only knowledge on the inputs and outputs (e.g. a grey box); ideally engineering designers would prefer to extend this knowledge and move to a white box where the interactions and the architecture of the interactions are known. Figure 3 graphically summarises this idea. In the grey box, on the left side of the figure, an organ has been selected to implement a function. This organ is associated with variables and metrics but the architecture and the interactions have not been defined. The right part of the figure represents a white box where the architecture and interactions have 
been defined. Developing the linked transformation process is the goal of the following section of this work.

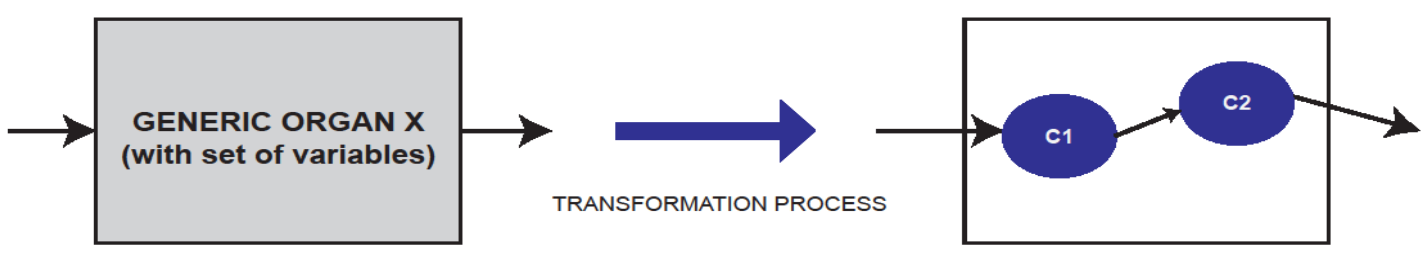

Fig. 3. Representation of the behaviour model of an organ, $\mathrm{C} 1$ and $\mathrm{C} 2$ represent clusters of descriptive variables.

The aim of the proposed approach is to provide support in decision making for the design teams during the early stages of the design process. The support comes from possibility of evaluating and comparing different design concepts and their behavioural laws without needing to spend a large amount of time to gather knowledge related to these laws. The final aim of all decision support is to remove as much subjectivity as possible when making a choice as possible (Clemen, 1997), and, overall, multiple other approaches exist to further provide support in the decision making task although they do not tackle the issue of knowledge gathering. For example, the decision-matrix method introduced by Pugh (Pugh, 1991) allows the ranking of complex options in a set and is often used in engineering problems. Zwicky's work (Zwicky, 1969) with morphological analysis also focuses on the choice of relevant solutions; the method is especially interesting in the case of complex systems where no element should be dropped from the study.

\subsection{Moving from a list of variables to a model of the interrelation of these variables}

The present section develops a concrete approach helping transform an initial list of variables associated to a box in the Fig.3, and the associated metrics, into a model of the interactions between these variables. Such a model represents the behavioural law of the organ under consideration. Section 3 illustrates this approach with the case study of a beam structure.

The fundamental tool of the approach and making the transformation possible is the VaschyBuckingham theorem. This theorem is used for the metrisation of the classification space (Matz, 1959; Barenblatt, 1979; Sonin, 2001) and is presented below.

Let $y=\sum_{i} a_{i} x_{i}$ be a law. Then all $a_{i} x_{i}$ must have the same dimension as $y$. If $a_{i}$ is a dimensionless constant, then $x_{i}$ must have the same dimension as $y$. This is the principle of dimensional homogeneity. If the system of fundamental quantities needed to represent a specific law is in the form of three basic quantities, namely length $L$, mass $M$, and time $T$, and if $[y]$ is the dimension of the variables, it is then a combination of the three basic dimensions and has the form:

$$
[y]=C_{1} \cdot L^{\alpha_{1}} M^{\alpha_{2}} T^{\alpha_{3}}
$$

In this form, the constant $C_{1}$ and the exponents $\alpha_{1}, \alpha_{2}$ and $\alpha_{3}$ are dimensionless numbers. It follows from the product theorem that every law which takes the form $y_{0}=f\left(x_{1}, x_{2}, \ldots, x_{n}\right)$ can take the alternative form shown in Equation 2.

$$
\prod_{0}=f\left(\Pi_{1}, \Pi_{2}, \ldots, \Pi_{n}\right)
$$


$\Pi_{i}$ are dimensionless products and this alternative form is the final result of dimensional analysis. It is possible to represent any law between variables as the combination of dimensionless numbers. A dimensionless number is a product that takes the following form:

$$
\prod_{\mathrm{i}}=\mathrm{y}_{\mathrm{i}} \cdot\left(\mathrm{x}_{1}^{\alpha_{1}} \mathrm{x}_{2}^{\alpha_{\mathrm{i}_{2}}} \mathrm{x}_{3}^{\alpha_{\mathrm{i}_{3}}}\right)
$$

$\left\{x_{1}, x_{2}, x_{3}\right\}$ are repeating variables, $\left\{y_{1}, \ldots, y_{n}\right\}$ are performance variables (Bhashkar \& Nigam, 1990) and $\left\{\alpha_{i j} \mid 1 \leq i \leq n-r, x_{2}, x_{3}\right\}$ are exponents.

The Vaschy-Buckingham theorem, also known as the $\Pi$ theorem, is a universally used tool in the context of dimensional analysis. Nevertheless, it does not provide any specific guidance related to the two following choices: the selection of the repeating and performance variables, and the determination of the unique number of governing dimensions $\left(D_{\min }\right)$. That selection can be especially tricky in the case of complex problems and can lead to impasses; Section 3 includes an example of such a scenario.

An approach for making the appropriate choices has been proposed by Butterfield (Butterfield, 2001) and developed into an algorithm; it is summarized in Table 6. The approach can be elaborated as follows. $V$ is the list of independent variables assumed to govern the system. $R \in V$ contains the variables selected from $V$, which have distinct dimensions other than $0 . P$ are the variables not in $R$, which have been placed in this group because the dimensions of some of these variables repeat the dimensions of the variables in $R$. $O$ are variables which have zero dimension. $D$ is a possible set of $m$ independent variables of basic or composed dimensions. $Q$ is a set of variables selected from $R$, from which a dimensionless group cannot be formed. $Q$ forms the repeated variable list. The array $(m \times m)[A]$ is the outcome of the process of selecting variables to form dimensionless numbers; it should be checked that $[A]$ is non-singular (i.e. $\operatorname{det}(A) \neq 0$ or rank of $[A]$ is $m$ ). The number of components of $D$ to be $D_{\text {min }}$.

Table 6. Table for the selection of repeating and performance variables (adapted from Butterfield (2001))

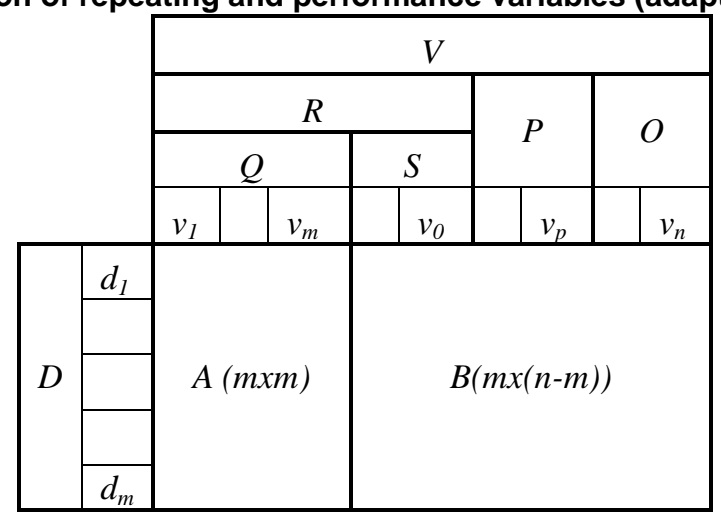

Nevertheless, Butterfield's approach remains unsatisfactory from an engineering point of view. Indeed, the list $Q$ is often not unique and consequently different models, all equivalent dimensionally, can be formed for the same problem. In order to avoid selecting a set of variables $Q$ that will lead to models that are inconsistent from engineering and scientific viewpoints, a simple heuristic can be applied. This heuristic states the following:

When several sets of variables can be selected in $Q$, the modeller should locate and choose the variables that he or she can influence and that are not imposed as constraints.

In practice, this means that if the length $L$ is imposed by some requirements to the designers, inertia $I$ is a variable that can be acted upon in the design, then $I$ should be selected over $L$.

The process presented above leads to the final formulation of the design problem in the form of a set of dimensionless numbers similar to the form of Equation 2. $\Pi_{0}$ is the dimensionless number containing the dependent variable of the problem and the other $\Pi$ numbers on the right side of the equation contain 
the problem's independent variables. After generating such an equation, the traditional approach, in qualitative physics, consists in using the machinery introduced by Bashkar and Nigam (Bashkar and Nigam, 1990) to reason qualitatively about the phenomenon under consideration. The goal of this work is different; it is to generate an approximated model of the phenomenon in order to simulate it quantitatively via a system dynamics approach. As such, this forms the novelty of the work and the next section presents the approach developed for the generation this approximated model.

\subsection{Generation of the approximated model}

In order to generate an approximated model of the phenomenon under consideration, there is a need for supplementary information to transform the dimensionless numbers in the form of Equation 2 resulting from the transformation process described in the previous section. The necessary information comes from a limited set of generic laws associated to organs. As shown in Figure 3, based on the work of Coatanéa (Coatanéa, 2005), a design solution can be associated with a functional vocabulary and its mapping through a generic organ. This means that if a solution to a design problem is a beam, and if this beam is associated to a function that is to support a mass, then the function and the solution can be mapped with a generic organ named $\mathrm{C}$-organ ( $\mathrm{C}$ for Capacitor). The generic organ (see Figure 3 ) is associated with the generic law:

$$
e_{2}=\frac{1}{C}\left(\int f_{1} \cdot d t+q(0)\right)
$$

In this law, $e_{2}$ is the effort, $f_{1}$ is the flow, $q$ is the momentum and $\frac{1}{C}$ groups all the other factors. In order to present and illustrate the approximation method, the example of the beam problem, further developed in Section 3, will be used. The variables describing this problem set in the form of Equation 2 result in:

$$
\frac{d}{I^{1 / 4}}=f\left(\frac{F_{1}}{E I^{1 / 2}} ; \frac{L}{I^{4}}\right)
$$

The effort $e_{2}$ in the generic equation is equivalent to the force $F_{1}$ in the dimensionless numbers in the right side of the equation. The flow $f_{1}$ corresponds to the displacement $d$ of the beam.

By simple inspection of the variables in Equation 5, the behavioural law of a beam, based on the generic law for a C-organ, can take the form:

$$
F_{1}=\frac{1}{C_{1}} d
$$

$C_{1}$ includes all the variables other than $F_{1}$ and $d$.

This generic form can then be transformed into Equation 7, which shares similarities with the form found in Equation 5.

$$
\frac{F_{1}}{C_{a}} \cdot C_{b}=\frac{d}{C_{c}}
$$

By inspecting and separating the dependent variable $d$ from the other variables forming the independent set of variables, it is possible to generate Equation 8, where $C$ is a dimensionless constant.

$$
C \frac{d}{I^{1 / 4}}=\frac{F_{1}}{E I^{1 / 2}} \cdot \frac{L}{I^{4}}
$$

The final form of the law then takes the form: 


$$
d=\frac{L}{C E I^{1 / 2}} F_{1}
$$

It should be noted that a similar principle can be applied to other generic organs listed by Coatanéa (Coatanéa, 2005). Furthermore, the method can be applied individually to single organs forming a more complex system and the system's behaviour can then be obtained by integrating the elementary laws together. To be able to integrate such elementary laws in a simple manner, this work proposes to use system dynamics as a modelling language. The next section presents the modelling principles to use in order to develop system dynamic models; in this article, the principles are applied at an elementary level but they remain the same for an application at a higher level.

\subsection{Generation of a system dynamics model}

The mapping principle between the elementary variables presented in Table 3 can be summarized using Table 7.

Table 7. Mapping between elementary variables and system dynamics basic bricks

\begin{tabular}{cccc}
\hline & & \multicolumn{2}{c}{ Stock } \\
& & & \\
\hline \hline Power variables & $\mathrm{X}$ & & \\
\hline Effort & $\mathrm{X}$ & & \\
Flow & & & $\mathrm{X}$ \\
\hline State variables & $\mathrm{X}$ & $\mathrm{X}$ \\
\hline Displacement & & $\mathrm{X}$ & \\
\hline $\begin{array}{c}\text { Connecting } \\
\text { variables }\end{array}$ & & & \\
\hline \hline
\end{tabular}

For simplifying the modelling, when either a stock or a converter can be used, the representation of a converter will be systematically selected. In practice, this means that in the example of a beam problem, displacement, momentum, and connecting variables such as Inertia (I) or Young modulus $(E)$ will always be modelled using converters.

The aim of a system dynamics model is to be simulated quantitatively. Thus, the user of the approach must select quantitative values, for the different variables, with appropriate units and magnitudes. Magnitude considerations will be added to the approach in future work.

\section{Beam Shape Selection Problem: Case Study Illustrating the Proposed Method}

The following case study does not discuss or include a description of the qualitative step that allows the transformation from a design problem to a design solution. The synthesis of solutions is not part of the contribution of this research work. Rather, the objective of this case study is limited to the demonstration that the practical framework developed in Section 2 can be used to gradually model design solutions. This framework provides the possibility of later developing a new type of support software for modelling and simulating design concepts during the early stages of the design process.

The present case study is organized in the following manner. A brief description of the design problem is followed by the presentation of three initial early solutions. All three belong to the same family of solutions to the problem and simply differ on the nature of their cross sections. A model of the solutions 
is then developed based on the step by step integration of the concepts presented in the previous section. This model is compared to a model widely available in literature and is implemented through a system dynamics approach. Finally, follows a discussion on the validity of the model and the possibility of applying the proposed approach to more complex problems.

\subsection{Description of the design problem}

The design problem consists in developing a technical solution able to support a heavy measuring device at a height of $3 \mathrm{~m}$ on the external wall of a building (Fig.4). All vertical movements of the measuring device should be minimised as it is supposed to be calibrated on a reference target on the adjacent wall. Furthermore, the ground below the measuring device should remain free of any kind of construction; therefore no support mast can be built. There is also an economic constraint; the final cost of the solution should be also minimized.

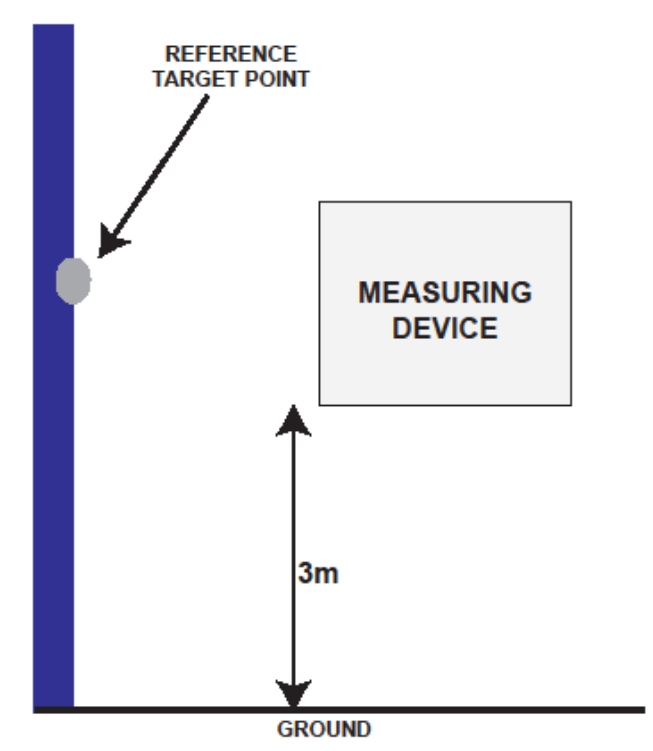

Fig. 4. Representation of the design problem.

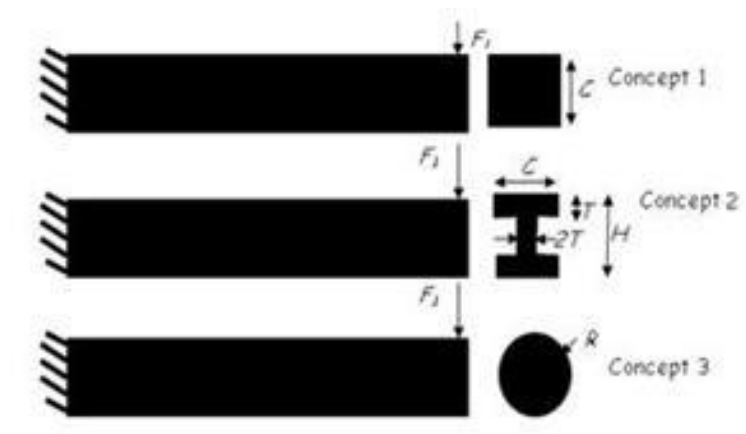

Fig. 5. Concepts of solution for a beam loaded with effort which causes bending.

It is supposed that the design team has developed three potential solutions, and, for simplification purposes, all solutions are considered to be based on the same principle of a beam. Future work will apply the similarity principle embedded in the first part of the proposed modelling technique (i.e. in the VaschyBuckingham theorem) in order to compare solutions based on radically different principles. 
Fig.5 illustrates the developed solutions as part of the early stages of a design process. They only differ by their cross sections and the aim is to compare them from the point of view of requirements. For this purpose, a step by step construction of the problem model is proposed below, based on the approach developed in Section 2. If supported by a computer tool, the need for experienced modellers would be greatly reduced and, thus, this aspect will be a main focus for future work.

\subsection{Progressive refinement to attain a model of the design solutions}

The beam can be represented (Fig.6) using a functional vocabulary based on the normalized vocabulary from Hirtz et al. (Hirtz et al., 2002). The main function of the beam is represented as a black box.

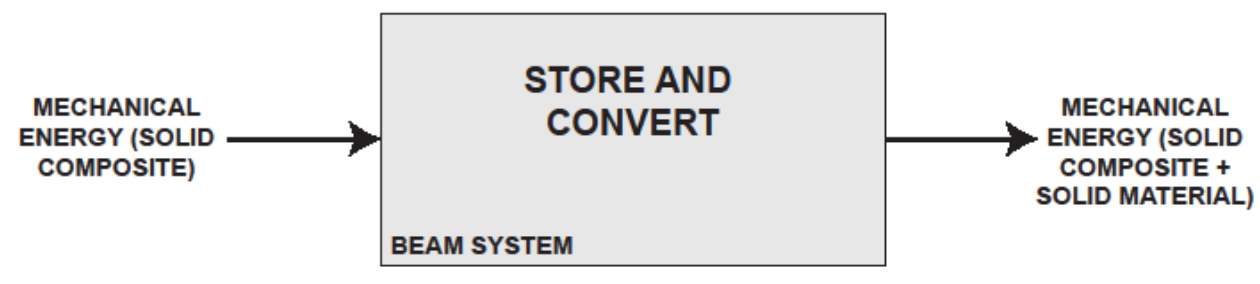

Fig. 6. Functional structure of a beam.

Once the functional description of the solution has been established, the potential generic organs that can be used to implement the function can be determined based on Table 4 . In the present case, a $C$-organ can be used. The selection should be made based on the functional vocabulary description but also the solutions that have been proposed. The selection of a generic organ indicates a list of elementary variables that should be used to describe this organ (see Tables 3 and 4). These variables are divided into three groups, power, state, and connecting variables. In the case of a beam, the taxonomy developed in Table 3 , and further in the work of Coatanéa (Coatanéa, 2005), indicates that it can be described using the state variable of displacement $d$ due to the bending effort and the connecting variables of the Young modulus $(E)$, Inertia $(I)$, and length of the beam $(L)$. Figure 7 shows the SADT diagram with these four variables and the input power variable $F_{1}$.

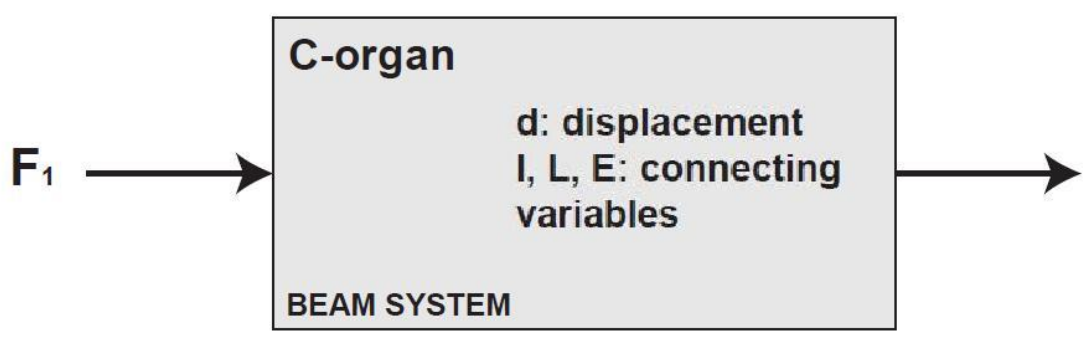

Fig. 7. SADT representation of the beam.

Table 8 summarises the list of variables selected as well as the metrics associated to these variables. According to the C-organ generic law, $A, I, L, d, F_{I}$, and $E$ are variables of interest for the analysis of how the proposed concepts can meet the requirements of supporting the mass of the measuring instrument while inhibiting the harmful effect (Altshuller, 1984) of bending. The three basic dimensions involved are $L, T$, and $M . d$ depends on the five other variables and while it is a complete set, as required, it is not an independent set. Indeed, once $A$ is specified, $I$ follows, and therefore one must be excluded. If $I$ is kept in the set, the following relationship expresses $d$ in terms of a complete set of independent variables: 


$$
d=f\left(I, L, F_{1}, E\right)
$$

Table 8. List of attributes and quantities involved in the problem

\begin{tabular}{|c|c|c|c|c|}
\hline \multirow[t]{2}{*}{ Functions } & \multirow[t]{2}{*}{ Comment } & \multicolumn{3}{|c|}{ Beam concept list of attributes and quantities } \\
\hline & & Power variables type & $\begin{array}{c}\text { Connecting and comparison } \\
\text { variable types }\end{array}$ & $\begin{array}{c}\text { State variables } \\
\text { type }\end{array}$ \\
\hline $\begin{array}{l}\text { STORE } \\
\text { and }\end{array}$ & $\begin{array}{c}\text { System } \\
\text { input }\end{array}$ & $\begin{array}{l}\text { Effort }\left(F_{l)} \text { in }\left(M L T^{2}\right)\right. \\
\text { velocity }\left(v_{l}\right) \text { in }\left(L T^{l}\right)\end{array}$ & & \\
\hline \multirow[t]{3}{*}{ CONVERT } & C-organ & & & $\begin{array}{c}d \text { bending } \\
\text { displacement } \\
\text { in } L\end{array}$ \\
\hline & & & $\begin{array}{l}A \text { section of the beam }\left(L^{2}\right) \\
I \text { inertia of the beam }\left(L^{4}\right) \\
L \text { Length of the beam }(L) \\
E \text { Young modulus }\left(M L^{-1} T^{2}\right)\end{array}$ & \\
\hline & $\begin{array}{l}\text { C-organ output } \\
\text { and I-organ input }\end{array}$ & $\begin{array}{c}\text { Effort }\left(F_{2}\right) \text { in }\left(M L T^{2}\right) \\
\text { Linear velocity }\left(v_{2}\right) \text { in }\left(L T^{l}\right)\end{array}$ & & \\
\hline
\end{tabular}

$d$ is the dependent variable and the set $I, L, F_{1}$ and $E$ are the independent variables. Note that the choice of a complete, independent set for this problem is not unique. There are four independent variables and one dependant variable, thus a total of five variables $(n)$; there are three base units $(m)$, and it is therefore theoretically possible to form $N=n-m=2$ dimensionless groups. Table 9 presents the clustering of variables based on the Butterflied algorithm (Butterfield, 2001). It is mainly used to select a valid set $Q x D$ ensuring that the matrix is not singular. The choice of the set $Q$ is not unique and, in this case, the choice has been made based on the application of the heuristic developed in Section 2. The full interest of this heuristic is shown at a later stage of the methodology.

Table 9. The first step of the selection of the performance and repeating variables

\begin{tabular}{|c|c|c|c|c|c|c|c|c|}
\hline & \multicolumn{7}{|l|}{$V$} \\
\hline & & \multicolumn{3}{|l|}{$R$} & \multirow{2}{*}{\multicolumn{3}{|c|}{$P$}} & $O$ \\
\hline & & \multicolumn{2}{|l|}{$Q$} & \multirow[t]{2}{*}{$S$} & & & & \\
\hline & & $E$ & $I$ & & $F_{1}$ & $d$ & $L$ & \\
\hline \multirow[t]{3}{*}{$D$} & $L$ & -1 & 4 & & 1 & 1 & 1 & \\
\hline & $T$ & -2 & 0 & & -2 & 0 & 0 & \\
\hline & $M$ & 1 & 0 & & 1 & 0 & 0 & \\
\hline
\end{tabular}

$Q$ is the set of repeating variables and $P$ forms the set of performance variables. The matrix formed by $Q x D$ is non-singular. According to Equation 3, is then possible to generate three dimensionless numbers, $\Pi_{1}, \Pi_{2}$ and $\Pi_{3}$.

$$
\left\{\begin{array}{c}
\Pi_{1}=F_{1} E^{-1} I^{-1 / 2} \\
\Pi_{2}=d I^{-1 / 4} \\
\Pi_{3}=L I^{-4}
\end{array}\right.
$$

According to the Vaschy-Buckingham theorem, the dimensionless numbers are related in the following manner: 


$$
\begin{gathered}
\Pi_{2}=f\left(\Pi_{1} ; \Pi_{3}\right) \\
\frac{d}{I^{I / 4}}=f\left(\frac{F_{1}}{E I^{I / 2}} ; \frac{L}{I^{4}}\right)
\end{gathered}
$$

The beam studied in this case study can be compared to a kind of spring than can be classified in the $C$-organ family of the Bond Graph theory (i.e. Capacitor organ). Such an organ is represented by a generic law shown in Equation 4. The bending, or behavioural, law of the beam takes the general form of Equation 6. Consequently, it is possible to consider that the combination of the three dimensional numbers of the problem takes the form shown in Equation 9, with $C$ being a dimensionless constant. Thus, the final form of the associated law is:

$$
F_{1}=C \frac{E I^{1 / 2}}{L} d
$$

If the goal is to calculate the displacement $d$ due to bending, the equation is easily transformed into Equation 10 as seen in Section 2.2.

For comparison, Equation 15 shows the law found in textbooks for such a beam problem, with $C_{1}$ being a constant.

$$
F_{1}=\frac{C_{1} E I}{L^{3}} d
$$

If, once again, the goal is to calculate the displacement due to bending, the following expression is obtained:

$$
d=\frac{L^{3}}{C_{1} E I} F_{1}
$$

Both Equations 14 and 15 (i.e. the reference equation) are valid from a dimensional point of view. Equation 14 is generated through the approach developed in Section 2. Equation 15 is the reference equation found in literature (Boresi, et al., 1993). When comparing the two equations, it is possible to see that Equation 14, and therefore also Equation 10, minimises the negative effect of the length of the beam on the increase of the displacement. Nevertheless, the impact of the length on the displacement can still be evaluated using this approximated form. Moreover, the two laws are comparable in terms of taking into account the impact of a variation in the shape of the cross section and, therefore, it can perfectly be evaluated with the proposed approach. Consequently, it can be concluded from this short analysis that a novice designer can use the approximated law of the bending of the beam to support the selection of a design solution.

Table 10 summarises the results from this initial modelling stage. The results are based on the hypotheses that the mass of the measuring device $e$ is $2000 \mathrm{~kg}$, the gravitational constant is $9.81 \mathrm{~m} / \mathrm{s}^{2}$, both $C$ and $C_{l}$ are taken equal to 1 as they do not affect decision making at this stage and the Young modulus is in the range of $200 \mathrm{GPa}$ (approximately the value for steel).

It should be noted that, at this stage, it is possible to see that a permutation between the two variables $I$ and $L$ in the sets $Q$ and $P$ of Table 8 leads to the following law:

$$
d=\frac{I}{C_{1} E L^{5}} F_{1}
$$

Such a law shows that an increase in the length of a beam limits its bending while an increase in inertia increases it. The opposite is obviously correct. Therefore, the example of this permutation tends to demonstrate that the heuristic presented in Section 2 supports the development of approximated laws respecting physics and reference laws. 
Table 10. Evaluation of the different concepts of solutions using the approximated law and the reference law

\begin{tabular}{|c|c|c|c|c|c|c|c|c|}
\hline & Figure & & Dimensions (m) & $\operatorname{Section}(\mathbf{A})\left(\mathrm{m}^{2}\right)$ & Inertia(I)(m & $\mathbf{d}_{\text {evaluated }}(\mathbf{m})$ & $d_{\text {ref }}(m)$ & \\
\hline Concept 1 & & $\mathrm{C}$ & 0.45 & 0.2 & 3,33E-03 & $1.77 \mathrm{E}-04$ & $7.95 \mathrm{E}-04$ & \\
\hline \multirow{3}{*}{ Concept 2} & & $\mathrm{C}$ & 0.45 & & & & & \multirow{3}{*}{$4 \mid \begin{array}{c}\text { Selected } \\
\text { concept } \\
\text { of } \\
\text { solution }\end{array}$} \\
\hline & & $\mathrm{H}$ & 0.45 & 0.2 & $3.42 \mathrm{E}-03$ & $1.72 \mathrm{E}-04$ & $7.75 \mathrm{E}-04$ & \\
\hline & & $\mathrm{T}$ & 0.20 & & & & & \\
\hline Concept 3 & & $\mathrm{R}$ & 0.25 & 0.2 & $3.18 \mathrm{E}-03$ & $1.85 \mathrm{E}-04$ & $8.32 \mathrm{E}-04$ & \\
\hline
\end{tabular}

The next step consists in modelling the approximated law in system dynamics language. Fig. 8 depicts the form of the model used.

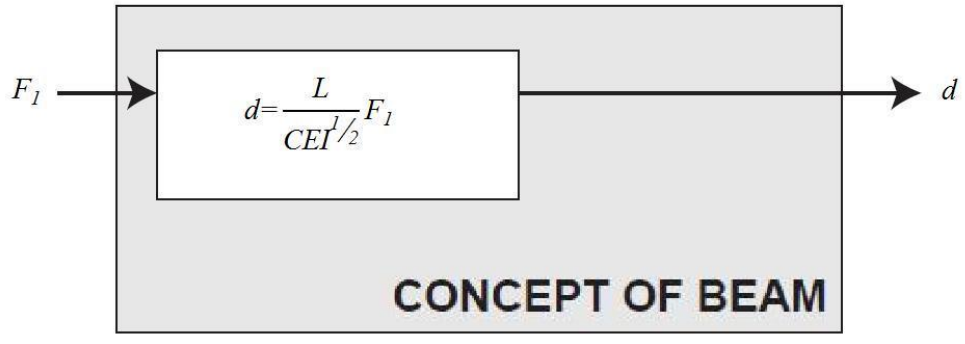

Fig. 8. Representation of the law of the beam.

The mapping between the variables of the approximated law and the components of a system dynamics model can be done according to Table 11. In short, power variables are mapped with flow in system dynamics, while state and connecting variables can be mapped either with stocks or converters. Nevertheless, as indicated in Section 2.3, for modelling simplicity, state and connecting variables are systematically mapped with converters. Indeed, a mapping with stocks greatly limits modelling as stocks only accept connections from flows located before or after them in a given model.

In the case of a beam problem, an approximated law has been established above. The modelling is done by systematically selecting converters over stocks for state variables. Using a system dynamics approach, the influence of any of the independent variables can be simulated. As there is dedicated software, this type of simulation is easily achieved and can provide useful information on the validation of each variable. In the rest of the case study, the variable simulated is inertia $I$. 
Table 11. Mapping between beam problem variables and SD components

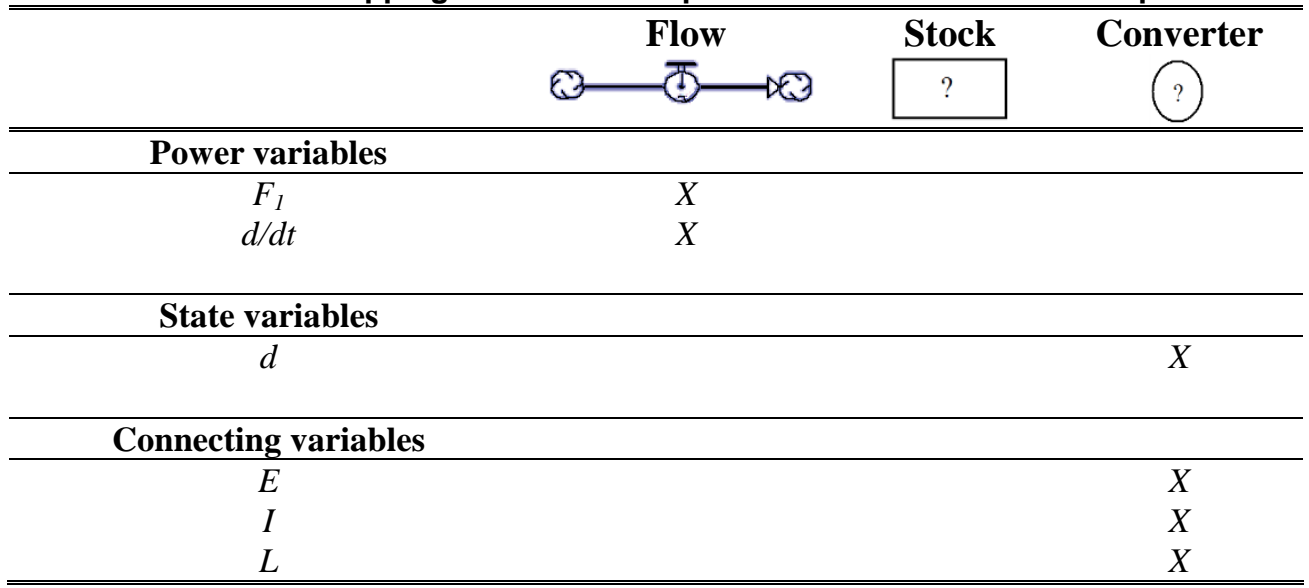

Fig.9a depicts the systems dynamics model obtained with the mapping proposed in Table 11.

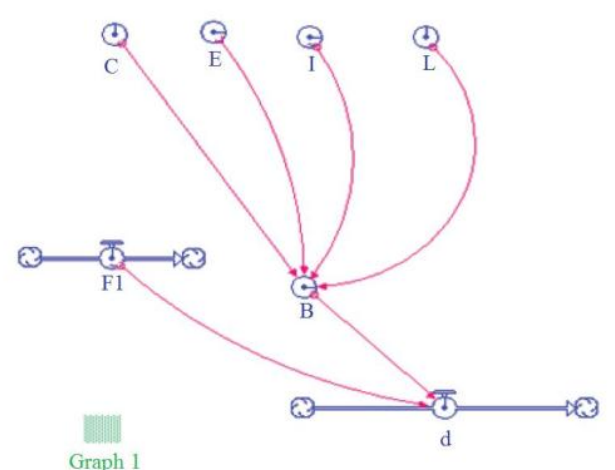

Graph 1

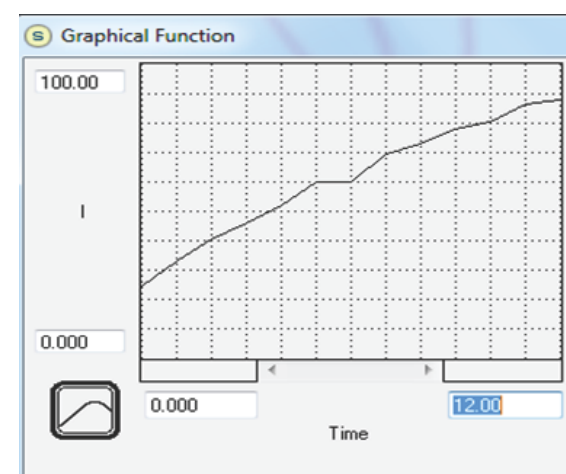

b.

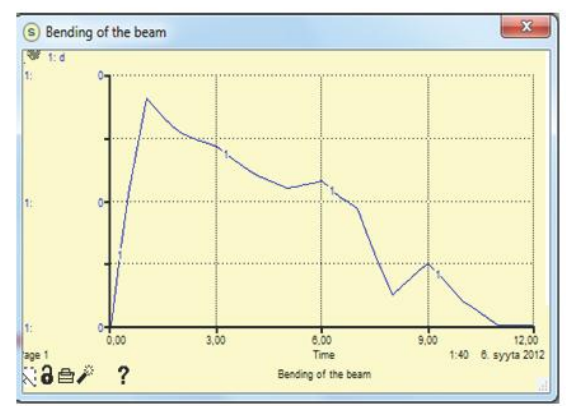

c.

Fig. 9. (a-c) System dynamics model and effect on the displacement due to dynamic changes in inertia.

Fig.9b is a representation of the dynamic input law for the inertia of the system and the resulting bending of the beam can be seen in Fig.9c. In practice, the variation in inertia may not be explicitly known but the simulation of the model (Fig.9a) allows the testing of different inertia properties and the evaluation of their impact on the displacement $d$ and therefore the bending of the beam.

Overall, the example of the displacement of a beam yields a simple model but it efficiently illustrates the approach developed in this research work. This approach will be taught in Master's level classes in the upcoming months in order to develop its usability. 


\section{Discussion - Conclusion}

The early stages of the design process highly influence the final outcome of projects but they are marked with immense time constraints, concept designs must be elaborated and evaluated in order to pursue the most promising ones. The design team cannot afford to waste time on seeking specific knowledge related to a design concept. The present article proposed an analytical approach that allows designers to evaluate and compare design concept solutions without seeking the specific behavioural laws that govern these concepts. The approach is based on multiple successive steps. First, the design activity is described in mathematical terms. Solutions are conceptualised as functions, organs, and mappings between these functions, organs, base metrics and units, forming "grey boxes". This conceptualisation leads to a cluster of variables and metrics that describe the problem. The second stage of the approach is to move away from a "grey box" to a "white box". The interrelations between the variables and metrics are modelled based on the Vaschy-Buckingham theorem, Butterfield's work, and a heuristic proposed by the authors. From this interrelation model, an approximated model, or behavioural law, is extracted. There is no need for any specific knowledge to obtain this law, the designer simple should model the concept and apply the proposed approach. As a final part of the work, the approximated law is modelled using system dynamics to better understand the dynamic influence of the different variables on the system. The application of the full approach is illustrated on the case of a beam displacement problem under bending conditions.

The contributions proposed in this work are as follows:

- A mathematical analysis of the early design process justifying the use, by engineering designers, of intermediate abstract concepts to move gradually from an initial description of needs to initial solutions,

- A practical method of combining these concepts for operational use,

- A method for modelling the behaviour of early design solutions and representing models in the form of a graph representation and a set of clustered descriptive attributes of the design problem,

- A demonstration of the method, with results that are in line with existing computational methods used for selecting the best cross section shape in the case of the bending of a beam.

The focus of the article has been on the evaluation and comparison of concept designs based on similar technological solutions. The implementation the comparison of concepts that use radically differing technological solutions is to be included in future versions of the approach. Indeed, the function fulfilled by each solution is the same and, at the early design stage, the input and output variables are similar or even identical. The behavioural laws will be different based on what is in the "box" (i.e. technological solution) but the influence of the input variables can be compared. In the future, the approach will also be included in software in order to make it easier to use and more accessible. Its further validation and applicability will also be tested in Master's level engineering design classes.

The mathematical bases for the approach have been largely discussed in Section 2; nevertheless, certain concepts should be explored further. The term "sufficiently detailed", used in the conditions necessary to form a metric space from a classification space, refers to the granularity level needed to reach in order to describe and study concept designs in a manner that would make their evaluation and comparison pertinent. This term is not analysed in mathematical terms in this article and remains to be tackled in future work.

The present work was centred on the notions of function and organ to describe design concepts, both are widely used and accepted in the engineering design field, as well as systems engineering. Nevertheless, certain authors claim that the concept of function does not grasp all the situations that can be encountered in artefact design. For example, some designs consider the notion of affordance, described as "action possibilities latent in the environment" by Gibson (Gibson, 1977), Norman (Norman, 1988), and it can present functions that were not intended by the original design team. Moreover, the evaluation of the approximated law with the proposed approach is done through tangible and quantifiable metrics. The framework reaches its limits when human perception comes into play. 


\section{References}

Altshuller, G. (1984). Creativity as an exact science. Luxembourg: Gordon \& Breach.

Barenblatt, G. I. (1979). Similarity, Self-Similarity and Intermediate Asymptotics. New York: Consultant Bureau Plenum.

Bhashkar, R., \& Nigam, A. (1990). Qualitative physics using dimensional analysis. Artificial Intelligence, 45(1-2), 73-111.

Boresi, A. P., Schmidt, R. J., \& Sidebottom, O. M. (1993). Advanced Mechanics of Materials. New York: John Wiley and Sons.

Bourbaki, N. (1966). General Topology (2nd ed.). Berlin: Springer-Verlag.

Butterfield, R. (2001). Dimensional Analysis Revisited. Proceedings of the Institution of Mechanical Engineers, Part C: Journal of Mechanical Engineering Science, 215(11), 1365-1375.

Clemen, R. T. (1997). Making Hard Decisions: An Introduction to Decision Analysis. Florence, KY: Duxbury Press.

Coatanéa, E. (2005). Conceptual Design of Life Cycle Design: A modeling and evaluation method based on analogies and dimensionless numbers (PhD Thesis). Helsinki University of Technology.

Cross, N. (2007). Forty years of design research. Design Studies, 28(1), 1-4.

Dardy, F., Teixido, C., Brissard, J. L., \& Polizzi, M. (2003). Guide de la compétitivité Industrielle: De la Conception à la Production. Paris: Delagrave.

French, M. J. (1999). Conceptual design for engineers (3rd ed.). Springer.

Gibson, J. J. (1977). The Theory of Affordances. In Perceiving, Acting, and Knowing: Toward an Ecological Psychology (pp. 67-82). Hillsdale, NJ: Lawrence Erlbaum.

Hirtz, J., Stone, R. B., McAdams, D. A., Szykman, S., \& Wood, K. L. (2002). A functional basis for engineering design: Reconciling and evolving previous efforts. Research in Engineering Design, 13(2), 65-82.

Hsu, W., \& Woon, I. (1998). Current Research in the Conceptual Design of Mechanical Products. Computer-Aided Design, 30(5), 377-389.

Lotter, B. (1986). Manufacturing Assembly Handbook. Boston: Butterworths.

Matz, W. (1959). Le principe de similitude en Génie Chimique. Paris: Dunod.

Miled, F. (2003). Contribution à une méthodologie de conception des systèmes dynamiques actifs (PhD thesis). University of Technology of Belfort-Montbéliard (UTBM).

Motte, D., Bjärnemo, R., \& Yannou, B. (2011). On the interaction between the engineering design and the development process models - Part I: Elaborations on the generally accepted process models. Proceedings of ICORD2011, Bangalore, India.

NASA. (2007). NASA Systems Engineering Handbook (NASA/SP-2007-6105, Rev 1). Washington, DC: NASA.

Norman, D. A. (1988). The Design of Everyday Things. New York: Doubleday.

Otto, K., \& Wood, K. (2001). Product Design: Techniques in reverse engineering and new product development. Upper Saddle River: Prentice Hall.

Pahl, G., \& Beitz, W. (1984). Engineering design: a systemic approach. London: Springer.

Pugh, S. (1991). Total design: integrated methods for succesfull product engineering. Addison-Wesley Pub.

Reich, Y. (1995). A critical review of General Design Theory. Research in Engineering, 7(1), 1-18.

Shim, T. (2002). Introduction to physical system modelling using bond graphs (lecture notes). University ofg Michigan-Dearborn.

Sonin, A. A. (2001). The physical basis of dimensional analysis (2nd ed.). Cambridge: Department of Mechanical Engineering MIT.

Stasinopoulos, P., Smith, M. H., Hargroves, K., \& Desha, C. (2009). Whole System Design: An Integrated Approach to Sustainable Engineering. London: Earthscan. 
Sterman, J. (2000). Business Dynamics: Systems Thinking and Modeling for a Complex World. Irwin/McGraw-Hill.

Sutherland, W. A. (1975). Introduction to Metric and Topological Spaces. Oxford: Oxford University Press.

Sydenham, P. H. (2004). Systems Approach to Engineering Design . Boston: Artech House .

Tomiyama, T., \& Yoshikawa, H. (1987). Extended General Design Theory. In H. Yoshikawa, \& E. Warman (Eds.), Design Theory for CAD. Amsterdam: North-Holland Publishing Company (pp. 95-130).

Top, J. L. (1993). Conceptual modelling of physical systems (PhD Thesis). Unvirsity of Twente.

Ulrich, K. T., \& Eppinger, S. D. (2000). Product Design and Development (2nd ed.). Mc Graw-Hill.

Yannou, B. (2000). Préconception de Produits - Mémoire d'habilitation à diriger des recherches (Discipline: Mécanique). Institut National Polytechnique de Grenoble (INPG).

Yoshikawa, H. (1981). General Design Theory and a CAD System. In T. Sata, \& E. Warman (Eds.), ManMachine Communication in CAD/CAM. Amsterdam: North-Holland Publishing Company (pp. 35-57).

Zeng, Y. (2008). Recursive Object Model (ROM) - Modeling of Linguistic Information in Engineering Design. Computers in Industry, 59(6), 612-625.

Zwicky, F. (1969). Discovery, Invention, Research - Through the Morphological Approach. Toronto: The Macmillian Company.

\section{Author Biographies}

Galina Medyna is a doctoral student at Aalto University - School of Engineering and expects to graduate in early 2013. Her research work, as well as her thesis, focuses on systems design considerations and environmental assessment based on exergy and dimensional analysis during the early stages of the design process.

Sarayut Nonsiri is is a doctoral student at Aalto University School of Engineering, Department of Engineering Design and Production. His research work is focuses on investigating in both theory and application for modeling and simulation during the early stages of the design process, model-based system engineering, computation algorithm, and artificial intelligence areas.

Eric Coatanea is a professor at Aalto University - School of Engineering; previously he was a researcher and teacher at the University of West Brittany. His research interests include design and manufacturing activities, system thinking and system engineering, eco-design, creativity and innovation. He created and leads the Early Development Process and System Engineering Research Group at Aalto University - School of Engineering. The group's research focus is the understanding and development of support tools for early design phases. The methods used to investigate this research domain include system thinking, system engineering, graph and network theory, natural language processing, computational linguistic and activity theory. During the past years, he has concentrated personal research efforts on problems discovery and clarification, problem solving, evaluation of design solutions and decision making process.

Alain Bernard graduated in 82, $\mathrm{PhD}$ in 89, was associate-Professor, from 90 to 96 in Centrale Paris. From Sept. 96 to Oct. 01, he was Professor in CRAN, Nancy I, in the "Integrated Design and Manufacturing" team. Since 0ct. 01, he has been Professor at Centrale Nantes and Dean for Research from 07 to 12. He is a researcher in IRCCyN laboratory (UMR CNRS 6597) in the "Systems Engineering -Products-Performances-Perceptions" team. His research topics are KM, PLM, information system modeling, interoperability, human safety modeling and evaluation, enterprise modeling, systems performance evaluation, virtual engineering, additive manufacturing, reverse engineering. He supervised $23 \mathrm{PhD}$ students, recently on extended enterprise modeling, simulation and performance evaluation. He published more than 250 papers in refereed international journals, books and conferences. He is chairman of WG5.1 of IFIP (Global Product Development for the whole product lifecycle) and chairman of CIRP STC Design. 